\title{
Hernia diafragmática congénita
}

\section{Congenital diaphragmatic hernia}

Jorge Isaac Ramírez Rivera ${ }^{1,2, a}$, Zoila Verónica Palacios Sagbay ${ }^{1,3, b}$.

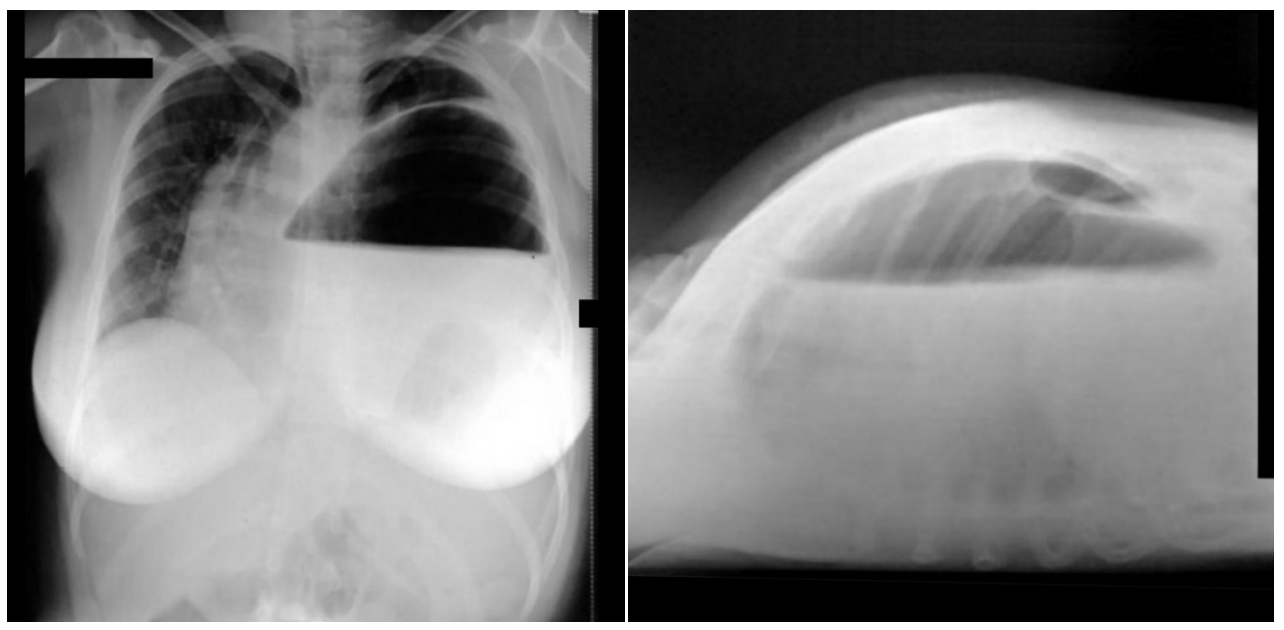

Adolescente mujer de 12 años con taquipnea. Sin historial de trauma, pero con antecedente de infección respiratoria frecuente desde los 2 años. A la auscultación se encontró el latido cardiaco desplazado hacia la derecha y ruidos hidroaéreos en el hemitórax izquierdo. Las radiografías antero posterior y lateral mostraron una imagen aérea ovoide con nivel líquido. En la laparotomía transversal izquierda se encontró defecto diafragmático posterior izquierdo o hernia de Bochdalek. Se logró reducir el estómago y colon transverso. El diafragma fue suturado y se colocó un tubo de tórax por $72 \mathrm{~h}$. La paciente egresó al día 7, con evolución favorable. La hernia diafragmática congénita de presentación tardía es infrecuente y muestra un cuadro con síntomas respiratorios o digestivos. El diagnóstico requiere alto nivel de sospecha clínica y es apoyado por medios de imagen, en este caso la radiografía de tórax.

A 12-year old female patient was admitted for tachypnea, there was no history of trauma but she remembered to have had frequent respiratory infections since she was 2 years old. Auscultation revealed displaced heartbeat to the right and abnormal respiratory sounds on the left hemithorax. Chest x-rays revealed an ovoid image with air and fluid content. A laparotomy revealed a left posterior diaphragmatic defect also called Bochdalek's hernia. The hernia was surgically repaired and a chest tube was inserted for 72 hours. The patient was discharged on day 7 uneventfully. Late onset diaphragmatic hernia is rare and usually presents with either respiratory or intestinal symptoms. A high index of suspicion is required supported by proper imaging.

\section{Correspondencia:}

Jorge Isaac Ramírez Rivera.

Correo electrónico: isaacramirezrivera@gmail.com

1. Servicio de Cirugía Pediátrica. Hospital del Niño Dr. Francisco de Icaza Bustamante. Guayaquil, Ecuador.

2. Universidad Internacional del Ecuador. Quito, Ecuador.

3. Universidad de Guayaquil. Guayaquil, Ecuador.

a. Cirujano Pediatra.

b. Médico. 